\title{
Package 'netassoc'
}

October 13, 2022

Type Package

Title Inference of Species Associations from Co-Occurrence Data

Version 0.7.0

Date 2022-07-20

Author Benjamin Blonder, Naia Morueta-Holme

Maintainer Benjamin Blonder <benjamin. blonder@berkeley. edu>

Description Infers species associations from community matrices. Uses local and (optional) regionalscale co-occurrence data by comparing observed partial correlation coefficients between species to those estimated from regional species distributions. Extends Gaussian graphical models to a null modeling framework. Provides interface to a variety of inverse covariance matrix estimation methods.

License GPL-3

Depends igraph, infotheo

Imports corpcor, huge, vegan

NeedsCompilation no

Repository CRAN

Date/Publication 2022-07-20 14:30:12 UTC

\section{R topics documented:}

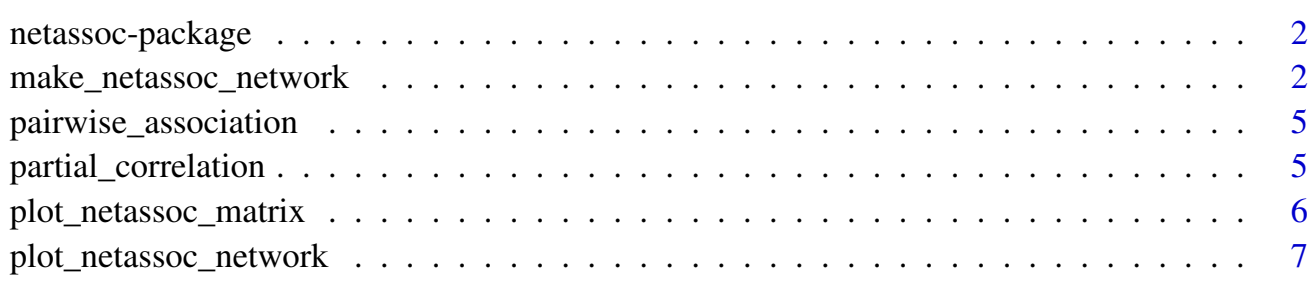

$\begin{array}{ll}\text { Index } & 10\end{array}$ 


\section{Description}

Infers species associations from community matrices. Uses local and (optional) regional-scale cooccurrence data by comparing observed partial correlation coefficients between species to those estimated from regional species distributions. Extends Gaussian graphical models to a null modeling framework. Provides interface to a variety of inverse covariance matrix estimation methods.

\section{Author(s)}

Benjamin Blonder, Naia Morueta-Holme

Maintainer: Benjamin Blonder <bblonder@gmail.com>

\section{References}

Morueta-Holme, N., Blonder, B., et al. A network approach for inferring species associations from co-occurrence data. (in review)

make_netassoc_network Infer species-association network

\section{Description}

Infers a species association network by determining which co-occurrence patterns between species are more or less likely than expected under a null model of community assembly. Defaults to estimation of association using a robust shrinkage estimator for inverse covariance matrices.

\section{Usage}

make_netassoc_network(obs, nul=vegan: : permatfull(obs)\$perm[[1]], method="partial_correlation", args=list (method="shrinkage", verbose=FALSE), p. method $=" f d r "$, alpha $=0.05$, numnulls $=1000$, plot=TRUE, plot.legend=TRUE, plot.title=TRUE, verbose=TRUE)

\section{Arguments}

obs

nul
A $m x n$ community matrix describing the abundance or presence/absence of $m$ species at $\mathrm{n}$ sites. Represents the observed data.

A $m \times n$ community matrix describing the abundance or presence/absence of $m$ species at $\mathrm{n}$ sites. Represents the regional null expectation data. The default value is a resampling of the observed data that preserves row and column sums, but this default method is not recommended. 
make_netassoc_network
method
The name of a function used to calculate relationships between species. The function must accept at least the arguments mat, a $\mathrm{m} \times \mathrm{n}$ (species $\mathrm{x}$ site) matrix. Defaults to partial_correlation.
args A list of additional arguments to be passed to the method function.
p.method The method used to correct $p$-values for multiple comparisons. See p.adjust for options.
alpha
Analysis-wide Type I error rate, controlled via the argument p. method.
numnulls
Number of resamples of the nul matrix used to assemble null communities. Larger values produce more accurate results.
plot If TRUE, plots all intermediate matrices calculated by the algorithm. Can be used to visualize input and output.
plot.title If TRUE, adds titles to diagnostic plots.
plot.legend If TRUE, adds legends to diagnostic plots.
verbose If TRUE, prints status updates and progress bars during calculations.

\section{Details}

Steps taken are:

1) obtaining input data and trimming to eliminate species that do not occur in any site 2) resampling a set of null community matrices from the expectation with the same richness and abundance as the observed community 3) calculating species co-occurrence scores for each pair of species within the observed matrix and all resampled null matrices 4) calculating standardized effect sizes and p-values for species' co-occurrence scores 5) thresholding effect sizes to retain only significant associations 6) converting matrix of scores to association network

The resulting network can be analyzed using functions from the igraph network package.

The user should specify a nul matrix of the same dimensionality as obs based on some regional distribution modeling approach (e.g. MaxEnt). The default reshuffling method is not recommended but provided to allow immediate output from the function.

This process by default builds a Gaussian graphical model via estimating an inverse covariance matrix (precision matrix, which can be used to calculate partial correlation coefficients) for all species pairs. This graph is then compared to a distribution of null graphs, such that the final output is a graph with edge weights corresponding to standardized effect sizes after correction for multiple comparisons.

A range of different methods are provided in partial_correlation for estimating relationships between species. Note that while a method is provided for the graphical lasso (L1-regularization) its use is not recommended, as it will produce very sparse null networks and then a narrow (or singular) distribution of null edge weights.

The inverse covariance methods implemented in partial_correlation result in symmetric association metrics. Non-symmetric metrics (e.g. describing predation or commensalism) are possible mathematically but their usage is not well-established. For an example of how to implement these, see pairwise_association. 


\section{Value}

A list with the following components:

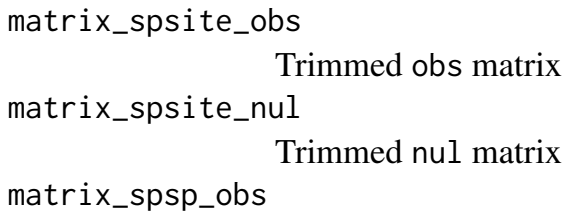

Observed co-occurrence scores for all species after removing those with nonsignificant p-values

matrix_spsp_pvalue

P-values for all species after correction for multiple comparisons

network_all An igraph object representing the association network

network_pos An igraph object representing an association network including only positive associations

network_pos An igraph object representing an association network including only negative associations

\section{See Also}

vegan::permat

\section{Examples}

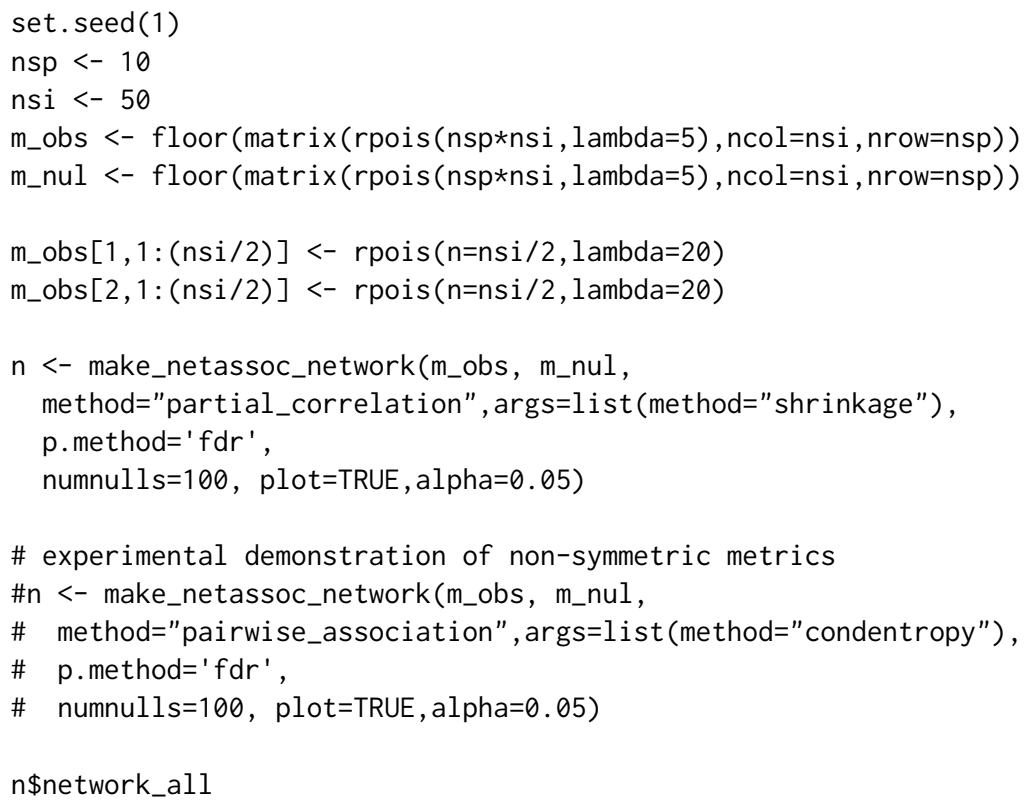


pairwise_association Pairwise associations

\section{Description}

Computes pairwise associations between every row (species) in a species $\mathrm{x}$ site matrix. Note that usage of this function is advantageous when non-symmetric association metrics are desired, but the pairwise computation will prevent accounting for indirect effects between species. As such this function should be considered preliminary, and its use experimental.

\section{Usage}

pairwise_association (mat, method = "condentropy")

\section{Arguments}

mat

method
A $\mathrm{m}$ x $\mathrm{n}$ (species $\mathrm{x}$ site) matrix

The name of a function to call to calculate an association score. Must take two vector arguments $(\mathrm{X}, \mathrm{Y})$ and return a single numeric value. Default argument uses conditional information entropy statistic, although other functions (e.g. Jaccard similarity) are possible.

\section{Value}

A n x n (species x species) matrix with NA diagonal values. May be non-symmetric depending on the method used.

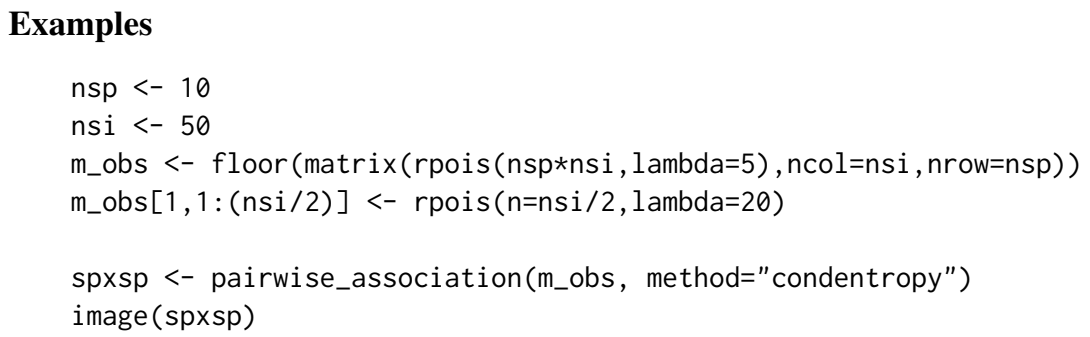

partial_correlation Partial correlation coefficients

\section{Description}

Estimates the inverse covariance matrix then uses this matrix to calculate partial correlation coefficents.

Assumes that matrix rows correspond to different variables of interest.

The one exception is if method="correlation"; see below for details. 


\section{Usage}

partial_correlation(mat, method, verbose=FALSE)

\section{Arguments}

Input matrix.

mathod

One of the following

"glasso" - L1-regularized estimation via graphical lasso. The analysis is run for a range of penalty parameters; then a single inverse covariance matrix is returned whose penalty parameter yields the with the best Extended Bayesian Information Criterion value. Uses huge: : huge and huge: : huge . select.

- "shrinkage" - Shrinkage estimate. The shrinkage parameter is estimated via an analytic formula. Uses corpcor: : invcov. shrink.

- "exact" - Directly calculates inverse covariance matrix. Results may be sensitive to highly collinear inputs. Use not recommended. Uses solve.

- "correlation" - Directly calculates correlation matrix. Does not return partial correlation coefficients as in all other cases. Use not recommended. Uses cor.

verbose Binary flag determining whether diagnostic output is shown.

\section{Value}

Returns a m x m upper triangular matrix of partial correlation coefficients from an input $\mathrm{m} \times \mathrm{n}$ matrix.

\section{Examples}

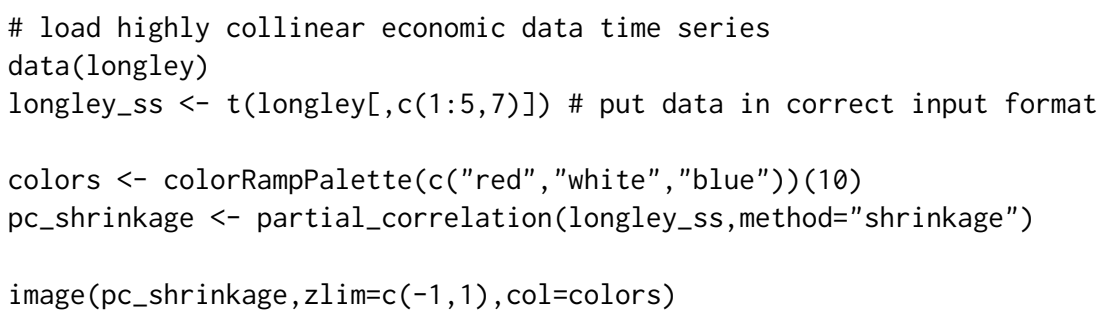

plot_netassoc_matrix Plots matrix with colormap

\section{Description}

Plots species $\mathrm{x}$ species or species $\mathrm{x}$ site matrix with color map 


\section{Usage}

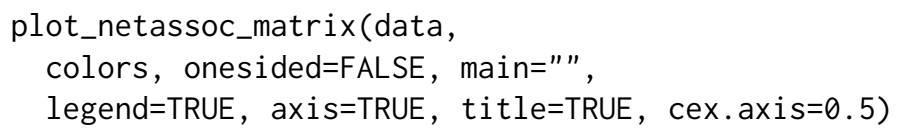

\section{Arguments}
data
Input matrix; assumed to have dimension names
colors
Vector of colors
onesided
If TRUE, assumes that colors maps to an entirely positive or entirely negative scale, i.e. without zero-crossings.
main
Title of plot.
legend
If TRUE, plots a colorbar legend.
axis
If TRUE, labels axes with dimension names.
title
If TRUE, plots a title.
cex.axis
Expansion factor for axis labels.

\section{Value}

None; used for the side effect of making a plot.

\section{Examples}

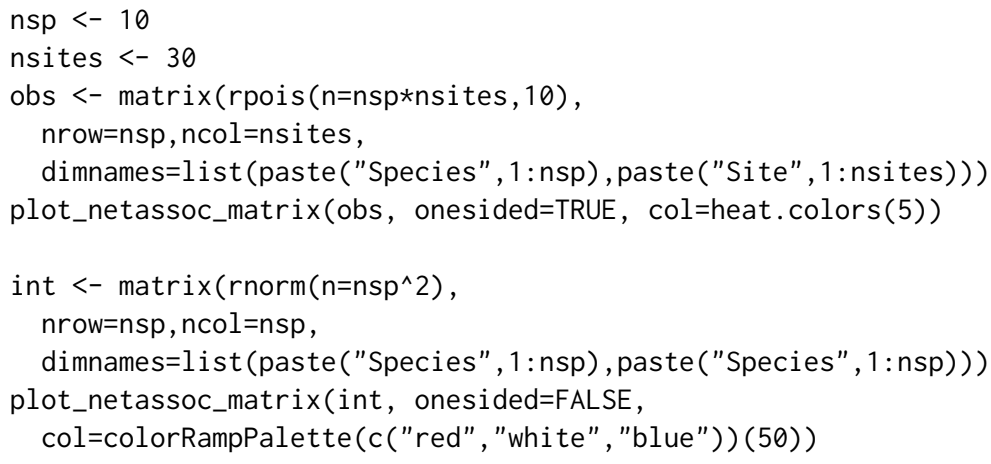

plot_netassoc_network Plots species association network

\section{Description}

Draws a network of species associations. By default edge widths are proportional to association strength and edge color reflects association type (blue, positive; red, negative). 


\section{Usage}

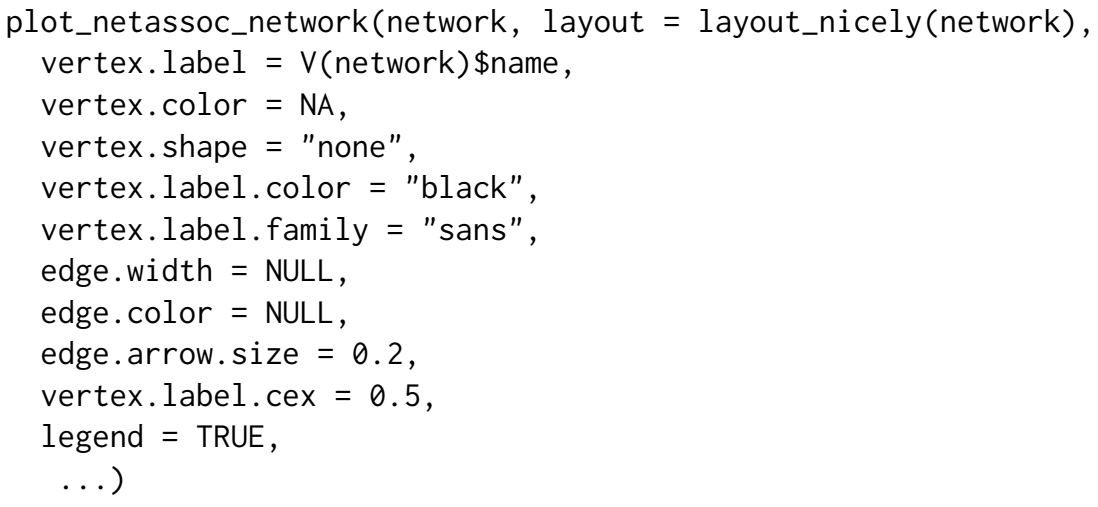

\section{Arguments}

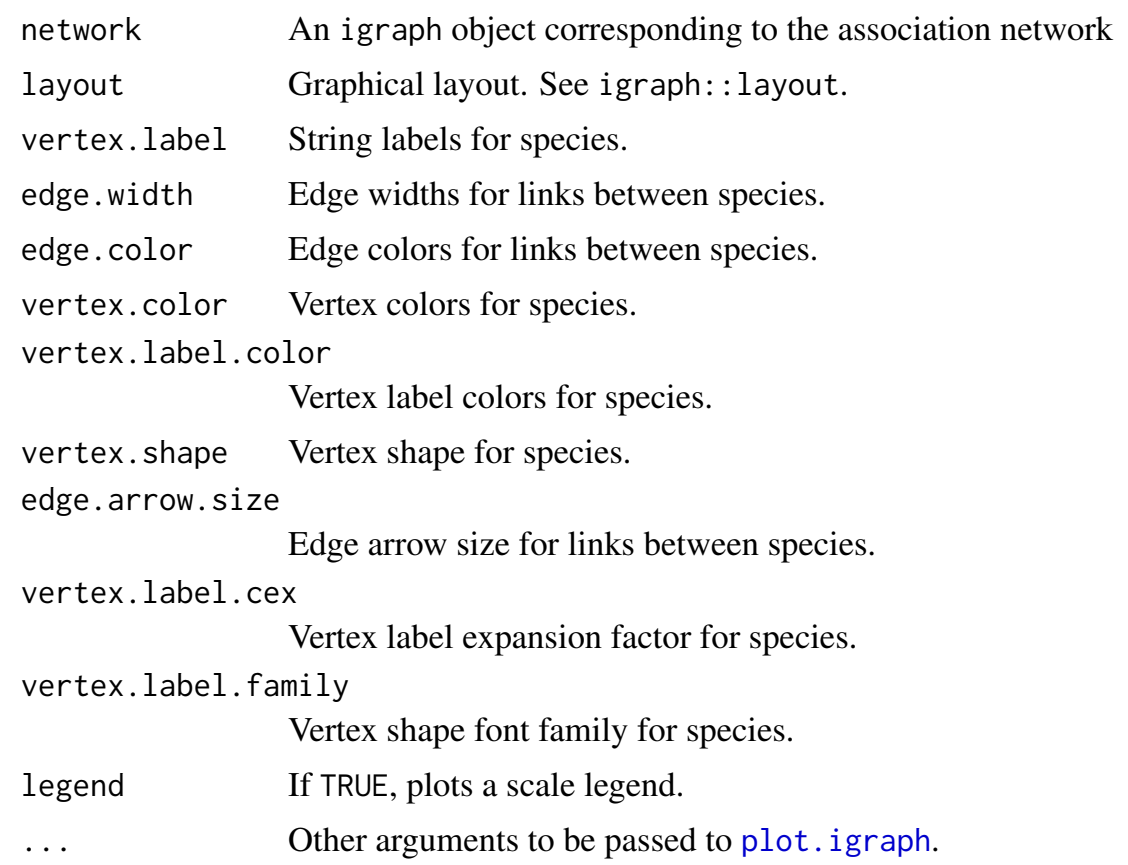

\section{Examples}

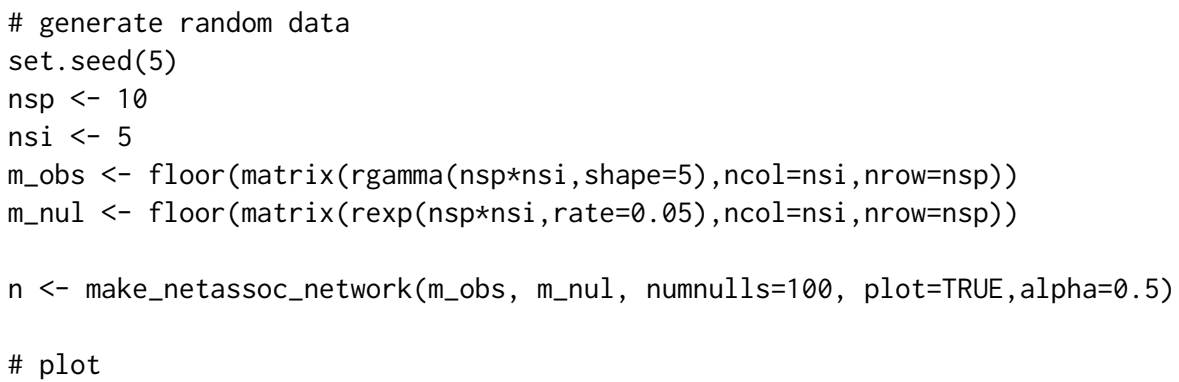


plot_netassoc_network

plot_netassoc_network(n\$network_all)

\# plot using circular layout

plot_netassoc_network(n\$network_all, layout=layout.circle(n\$network_all)) 


\title{
Index
}

\author{
* package \\ netassoc-package, 2 \\ make_netassoc_network, 2 \\ netassoc (netassoc-package), 2 \\ netassoc-package, 2 \\ p.adjust, 3 \\ pairwise_association, 3,5 \\ partial_correlation, 3,5 \\ plot.igraph, 8 \\ plot_netassoc_matrix, 6 \\ plot_netassoc_network, 7
}

